\title{
RESVERATROL AND ITS ANALOGUES - IS IT A NEW STRATEGY OF ANTICANCER THERAPY?
}

\author{
Sylwia BORYS ${ }^{1}$, Ronza KHOZMI ${ }^{1}$, Wiesława KRANC ${ }^{1}$, Artur BRYJA ${ }^{1}$, \\ Michal JESETA², Bartosz KEMPISTY ${ }^{1,3}$ \\ ${ }^{1}$ Department of Anatomy, Poznan University of Medical Sciences, Poznan, Poland \\ ${ }^{2}$ Department of Obstetrics and Gynecology, University Hospital and Masaryk \\ University, Brno, Czech Republic \\ ${ }^{3}$ Department of Histology and Embryology, Poznan University of Medical \\ Sciences, Poznan, Poland
}

DOI: $10.1515 / \mathrm{acb}-2017-0003$

Summary: Resveratrol (RSV) is one of the polyphenols - metabolites common in plants, however it does not occur in animals. It occurs mainly in grape skin (Vitisvinifera), peanuts (Arachis hypogeal) and in the roots of (Polygonumcupidatum) a traditional Chinese curative plant.

RSV has a preventive property against the most serious diseases of modern world such as cancer, neurodegenerative diseases and cardiovascular diseases. Due to pleiotropy, RSV is currently the main object of many research teams' interest, which is shown by the significant number of publications devoted to this subject.

Animal and human conducted studies have shown very low bioavailability of RSV (approx. 2\%), which is the result of rapid biotransformation to sulphate and to a lesser extent, to the glucuronide conjugates as well. The studies on the improvement of RSV bioavailability, which have beencarried out for many years, have contributed to the synthesis of the analogues of more chemopreventive and more desirable pharmacokinetic properties. In order to enhance antiproliferative activity and RSV bioavailability, series of methyl analogues were synthesized and this will be described later in more detail. An example of such a derivative is DMU-212 (3,4,4'5-tetramethoxystilbene)

Keywords: resveratrol, the methylated resveratrol analogues, apoptosis, cytotoxicity 


\section{INTRODUCTION}

From the second half of the twentieth century, there has been observance of the development of research on the composition and pro-health properties of wine and grapes. The history of research on Resveratrol has not been defined yet but it has roots in the early of eighties, when the Japanese researchers Arichi et al. (1982) noted that the dried roots Polygonumcuspidatum were used in traditional Chinese and Japanese medicine in the product called Kojo-kon. Itadori, also called Kojokon, was used to treat a wide range of ailments such as fungal infections, various skin inflammations or heart, blood vessels and liver diseases. Studies have shown that the main active ingredient Polygonumcuspidatum is RSV [45].

An observation has been contributed to the intensification of research on the composition of grapes and wine known as "French Paradox", which is a slogan that was formulated by French population. It shows that despite the lack of physical activity and a significant amount of consumed fats, along with wine consumption, there was an extremely low mortality rate for cardiovascular diseases [27].

\section{THE CHEMICAL STRUCTURE AND SOURCES OF RESVERATROL}

Resveratrol (3,5,4'-trihydroxy-trans-stilbene) is one of the best known stilbenoid polyphenols of natural origin. It exists as two stereoisomers. It is composed of two phenolic rings connected by ethylene bridge. In plants, it is formed in a reaction catalysed by one of thestilbenoid synthesis from one coumaroyl-CoA molecule and three malonyl-CoA molecules [43].

Under the influence of $\mathrm{UV}$ radiation, high temperature and $\mathrm{pH}$, the trans form is easily isomerised to cis stereoisomer. According to available literature, cis and trans isomers differ in biological activity and durability. The trans form, which occurs more commonly in natural raw materials, is more active and more durable. Other known stilbenoid polyphenols of natural origin consist of: piceatannol, pinosylvin, rapontigentin, pterostilbene, astrigin, piceid and viniferin [41, 35, 44, 13].

RSV is a phytoalexin produced by plants under stress factors such as bacterial and fungal infections, intense UV radiation, high temperature, nutrient deficiencies and mechanical damage. RSV was found in 72 different plants. Black grapes are the best source of 3,5,4'-trihydroxy-trans-stilbene and the red ones contain more of it than the green ones. Red wine also contains large amount of RSV that has been released from grape skin in fermentation process. Berries are the other natural source of RSV. Such a compound is also present in the leaves and flowers of Scots pine, white hellebore, orchids and rheum. 3,5,4'-trihydroxy-trans-stilbene was also found in cocoa, tomato skin and chocolate $[43,12]$. 


\section{THE BIOLOGICAL ACTIVITY OF RESVERATROL}

In view of the multidirectional RSV properties, research on the biological activity has been carried out by many research groups over the past decade. RSV has a preventive property against the most serious diseases of modern world such as cancer, neurodegenerative diseases and cardiovascular diseases. Furthermore, RSV delays ageing and have moderate antibacterial and antifungal properties $[46,17]$.

RSV inhibits the carcinogenesis by affecting the initiation process, promotion and progression of cancer. In tests carried out in both in vitro and in vivo models, the ability of RSV to inhibit cancer by multiple mechanisms has been demonstrated. The results of tests carried out on male rats dosed with $20 \mathrm{mg} / \mathrm{kg}$ of RSV for 28 days show that RSV does not have any significant systemic toxicity. Such a dose is a thousand times higher in comparison to the daily dose consumed by humans $[46,18]$.

Confirmatory tests proving the RSV chemopreventive properties, which have been conducted in the last five years, primarily focus on lung, breast, prostate and colon cancer models.

Moreover, RSV shows anti-diabetic properties and protective effects on diabetic complications. Positive preventive actions in diabetic neuropathy is the result of nuclear factor erythroid 2-related factor2 (Nfr2), SIRT1 (NADdepent protein deacetylase sirtuin-1) activation and the inhibition of NF- $\kappa B$ (Nuclear factor- $\mathrm{kB}$ ) and AP-1 (Activator protein 1) activation. SIRT1 is an important molecular regulator of energy metabolism and cellular mitochondrial homeostasis. Therefore, the activation of SIRT1 is an alternative way to improve the function of mitochondria [38].

Several studies, using both in vitro and in vivo model, have shown neuroprotective properties of RSV. In cell culture isolated from the infant rat's midbrain, which had stroke,there has been observance of reduction of the reactive oxygen from Reactive Oxygen Species (ROS) and a significant increase in the level of extracellular glutathione as a result of RSV [26]. In the observed test group of rodents with models of stroke, a reduced stroke impact area and improved cognitive functions after parenteral and oral intake of RSV were observed. Best neuroprotective effects of RSV occur when the intake took place during or immediately after stroke [40]. RSV has also a positive effect in Alzheimer's disease because of its ability to reduce cognitive disorders, interneural amyloid beta deposit, hyperphosphorylation of tau protein in the hippocampus and its neuroprotective properties. In behavioural studies of mice, there has been demonstrated that RSV improves memory by SIRT1-dependent pathway [29].

As evidenced by the results of numerous studies, RSV shows anti-inflammatory properties. Rahal et al.[33] have shown that RSV in a rat model of Crohn's disease reduces the level of inflammatory cytokines (TNF- $\alpha$, TNF- $\beta 1$ oraz IL-1 $\beta \mathrm{i}$ IL-6) and decreases the level of tissue fibrosis. In a model of acute pancreatitis induced 
by taurocholate, RSV reduced histopathological changes and lowered the level of biochemical markers in blood serum (renin activity, angiotensin II level, endothelin and NO) [5].

In literature, there is much information about the therapeutic effects of RSV on cardiovascular system diseases. During the studies conducted on rats with spontaneous hypertension, there has been shown that the treatment with RSV reduces systolic blood pressure, cardiac fibrosis and it increases the efficiency of its functions. RSV reduces the area of myocardial infarction and reperfusion time in rats [29]. As it has been demonstrated in the tests with mice with diet induced hypercholesterolemia, RSV has protective effects on atherosclerosis. The test group showed a decrease in aortic area changed by atheromatous and in cholesterol level in blood plasma, an increase in number of stability markers of atherosclerotic plaque and improvement of macrophage function [4].

In addition to anticancer, neuro and cardiovascular protective effects, RVS shows the ability to retard the cells ageing through the increase in the activity of telomerase. In tests carried out on older (14-15 months old) female mice after a year-long RSV supplementation, in addition to the increase in the activity of telomerase, there was also an increase in the length of telomeres and gene expression related to SIRT1. Increased expression of SIRT1, resulted in higher telomerase activity. Basing on the above-mentioned study results, it can be concluded that there occurs a positive knock-on effect between the gene expression of SIRT 1 and telomerase activity [23].

RSV and its metabolites have an influence on the expression of various isoforms of cytochrome P450. Not only does 3,5,4'-trihydroxy-trans-stilbene have a moderate ability to inhibit CYP1A1 and CYP1B1 but it has also poor ability to inactivate CYP1A2. The research conducted by Mikstacka et al. [25] have presented that RSV methyl derivatives show greater selectivity and inhibition of cytochrome P450(CYP) than RSV.

In the above-mentioned available literature, data shows that RSV is a molecule which has multidirectional pharmacological activity. Moreover, a very low RSV bioavailability limits the use of its healing potential. Conducted research on the RSV analogues synthesis, including its methyl derivatives, are intended to obtain compounds with improved pharmacokinetic and pharmacological properties.

\section{THE BIOAVAILABILITY AND BIOTRANSFORMATION OF RESVERATROL}

RSV contained in red wine has the highest bioavailability, however, it is much lower in white wine and grape juice [6,30]. In human body, RSV is absorbed in jejunum and ileum. Animal and human conducted studies have shown very low bioavailability of RSV (approx. 2\%), which is the result of rapid biotransformation 
to sulphate and, to a lesser extent, to the glucuronide conjugates as well. RSV sulfate derivatives appear in the bloodstream in about 30 min after an intake. Such compounds are detectable in the blood up to 9 hours after an intake and then they are excreted in urine and feces. The presence of P450 1A2 inhibitors results in a significant reduction in the number of created metabolites. After an intake, RSV is metabolised in hepatocytes and its $\mathrm{t}_{0.5}$ ranges from 8 to 14 minutes. RSV is a substrate for three cytochrome P450 isoforms: CYP1A1, CYP1A2 and CYP1B1.

CYP1B1 is involved in the RSV metabolic activation by its hydroxylation to piceatanool (3,5,4'-trihydroxy-trans-stilbene), which has a strong cytotoxic activity against cancer cells. Potter et al. [32] have demonstrated the occurance of three metabolites formed by the hydroxylation of RSV tetrahydroxystilbene (piceatanool), pentahydroxysystillbene and tetrahydroxystilbene. The largest amount was present in piceatanool. Piceatanool inhibits CYP1B1 activity but it does not inactivate the enzyme completely.

In vitro model studies have shown that $\mathrm{RSV}$ is an inhibitor of $\mathrm{P} 4501 \mathrm{~B} 1$ isoform (procarcinogenic activator) in human bronchial epithelial and breast glandular epithelium cells [42]. In vitro studies have demonstrated the ability of RSV to irreversible inhibition with Ki of $20 \mu \mathrm{M}$. Furthermore, Yu et al. [47] have reported that RSV and its metabolite (trans-resveratrol-3-O-sulfate) are CYP3A inhibitors. Chang and Yeung [9] have shown that RSV inactivates CYP3A4 in the presence of NADPH. The wide scope of RSV activity on human body, both in prevention and treatment of various diseases, is limited by its extremely low bioavailability (approx. 2\%). The studies on the improvement of RSV bioavailability, which have been carried out for many years, have contributed to the synthesis of the analogues of more chemopreventive and more desirable pharmacokinetic properties [16].

\section{THE RESVERATROL ANALOGUES}

The following elements of a compound chemical structure have the essential meaning for the biological activity of RSV and its derivatives; the number and position of hydroxyl groups, the presence of intramolecular hydrogen bonds, spatial isomerism and double bonds [28].

The analysis of the relationship between chemical structure and biological activity has demonstrated that the replacement of the hydroxyl groups on methyl enhances the cytotoxic effects of created RSV derivatives. In order to enhance antiproliferative activity and RSV bioavailability, series of methyl analogues were synthesized and this will be described later in more detail [15].

Furthermore, Colin et al. [11] have demonstrated that the acetyl RSV derivatives are captured more efficiently by the cell as compared to the parent compound with the maintenance of the cytostatic and cytotoxic effects on the starting material level. 
Another important observation of the relationship between chemical structure and biological activity is the fact that the ability to inhibit the growth of human prostate cancer cells DU-145 by RSV derivatives is maintained or even increased in the case of the absence of free hydroxyl groups. It is worth noticing that the results of the earlier published works indicate that the presence of free hydroxyl groups is required to inhibit the cell cycle and to reduce DU-145 cell lines lifespan [8]. Regev-Shoshani et al. [34] have described the effects of methylation and RSV glycosylation at p-hydroxy or m-hydroxy position. These modifications increase the protection against enzymatic oxidation and they prolong its half-life in the cell.

\section{THE METHYLATED RESVERATROL ANALOGUES}

RSV methyl analogues have similar biological activity to the parent compound. They are characterized by increased bioavailability due to enhanced lipophilicity so that they are transported into cells more easily. RSV methylation also increases immunity to biotransformation and inhibits the metabolism rate [19].

Cardile et al. [7] have shown that the substitution of phenyl stilbene ring for methyl group at position 3,4 and 4,3,5 enhances anticancer properties of created derivatives. The biggest biological activity of the tested compounds was found in a natural RSV analogue: 3,4', 5-trimetoxystilbene (TMS) which shows GI $_{50}$ (concentration of the anticancer drug that inhibits cancer cell growth by $50 \%$ ) on vinorelbine level.

TMS, comparing to RSV, has stronger anti-proliferative properties in several cell lines such as: SKOV-3 (ovarian cancer), Caco-2 (colon cancer), A-549 (lung cancer), Hep-2 (liver cancer), Vero (kidney cancer). The intensification of TMS anticancer property is likely to stem from two characteristics that distinguish it from RSV. Three TMS methyl groups significantly increase the lipophilic character of the molecule, which results in increased cell membrane permeability and in effect causes an increase in the intracellular concentration of TMS. Furthermore, the results of the conducted studies indicate that TMS has the ability to destabilise the process of microtubules in cancer cells at very low concentrations such as $1 \mu \mathrm{M}$ RSV, in contrast to TMS, does not show such properties [1].

Studies conducted with the annexin V assay and morphological tests on the acute myeloid leukemia (HL-60) cell lines demonstrate that the pro-apoptotic effect induced by TMS is 10-12 times higher as compared to RSV [39].

Belleri et al. [3] have demonstrated that antiangiogenic TMS, defined by the endothelial cell proliferation and morphogenesis, was a 100 times stronger than that of RSV which constituted the starting material. Furthermore, it has been shown that TMS has stronger anti-inflammatory and anti-atherosclerotic 
activity. The mechanism of such an action involves the inhibition of inflammatory cell adhesion and the activation of NF-kB channel [14]. The results of these studies show that after intravenous administration to rats a single dose of RSV $(5 \mathrm{mg} / \mathrm{kg})$, the clearance was 8-9 times higher than for TMS, which indicates more rapid RSV elimination from the body [22].

\section{DMU-212}

A conducted research in both in vitro and in vivo models have already showed stronger anti-proliferative and proapoptotic activity and greater bioavailability of RSV derivatives with ortho-methyl substituents as compared to a parent compound. An example of such a derivative is DMU-212 (3,4,4'5-tetramethoxystilbene) [10].

The results of the tests conducted by Sale et al. [37] point out an increased DMU212 bioavailability in the small intestine, colonic mucosa and brain as compared to RSV. Higher DMU 212 availability in brain suggests that it overcomes the blood-brain barrier more easily than RSV. Such a change in the pharmacokinetics of a compound may be the result of an increase in DMU-212 lipophilicity, which happaned as a result of replacing three hydroxyl groups present in the RSV with four methyl groups.

Such a discrepancy in the metabolic profile is another factor which determines the differences in the pharmacokinetic features of RSV and DMU-212. As it is known, RSV undergoes phase II metabolic reactions which means conjugation with glucuronic acid and sulfuric acid. DMU-212, however, undergoes oxidation in liver, primarily O-demethylation at positions 3,4 or 4 ' and hydroxylation. As a result of DMU-212 biotransformation, there are produced five major metabolites: DMU-295 (4,4'-dihydroxy-3,5-dimethoxystilbene), DMU-281 (4'-hydroxy-3,4,5-trimethoxysytilbene), DMU-214 (3'-hydroxy-3,4,5,4'tetramethoxystilbene), DMU-291 (4-hydroxy-3,5,4'-trimethoxystilbene), DMU807 (3-hydroxy-4,5,4'-trimethoxystilbene).

Sale et al. [37] have demonstrated that methylation of hydroxyl groups with RSV and the introduction of new methyl group in position 4 destroys the ability to inhibit COX-2 expression. However, it does not limit the potential for inhibiting the adenomas formation and the ability to reduce PGE-2 level.

The results of studies conducted by Cichocki et al. [10] on ovarian cancer cell culture have shown that DMU-212 has stronger antiproliferative and apoptotic effect than RSV. Other groups have received similar test results on different cell lines. DMU-212 also show anti-inflammatory activity as a result of pro-inflammatory transcription factors inhibition particularly NF-kB and iNOS (inducible nitric oxide synthase), which has been confirmed in a model of rat liver 
cancer. The achieved effects make DMU-212 a good starting point for designing compounds with greater anti-proliferative activity.

DMU-212 shows more potent anticancer properties than RSV respectively in human breast cancer cell lines MDA-MB-435 and MCF-7. The mechanism of this action may occur due to the cell cycle arrest in G2/M phase by RSV. Moreover, DMU212 induces apoptosis by reducing the expression of anti-apoptotic proteins Bcl-2 and Bcl-XL. DMU-212, similarly as RSV, also inhibits cell growth by inhibiting Stat 3 protein activation which has oncogenic properties. On the contrary to RSV, DMU-212 causes a significant increase in tubulin polymerisation, which indicates the validity of research on DMU-212 to demonstrate its antimitotic activity [24].

Cytochrome P450 CYP1B1 isoform is overexpressed in a variety of human cancer types (e.g. breast, prostate, uterus and ovaries). Isoforms CYP1B1 and CYP1A 1 have the ability to activate many procarcinogens and catalyse the estrogen hydroxylation, which seems to be a key element of the hormone-dependent carcinogenesis. That is why the compounds with the ability to inhibit CYP1A1 and CYP1B1 protein expression may represent a new class of potential anticancer drugs. Piotrowska et al.[31] have showed that DMU-212 reduces CYP1A1 and CYP1B1 protein expression in human ovarian cancer cell lines SKOV-3 and A-2780. In the case of cell line A-2780, there was reported a total inhibition of CYP1B1 protein expression. The results of this study also indicate that DMU-212 has the ability to regulate genes which are responsible for the process of apoptosis in both mitochondrial and extrinsic pathways.

\section{THE ANALOGUES OF DMU-212}

In vivo metabolic demethylation and hydroxylation lead to the creation of five metabolites DMU-212: DMU-214, DMU-281, DMU-291, DMU-295 and DMU807 which have potential cytotoxic properties [37].

Androutsopoulos et al.[2] have demonstrated that DMU-214, similarly as a parent compound, shows cytotoxic properties in submicromolar concentrations against cell lines breast MCF-7 and liver cancer HepG2. The inhibition of cell proliferation by DMU-214 occurs as a result of apoptosis induction - inhibiting cell cycle in $\mathrm{G} 2 / \mathrm{M}$ phase, decreasing p53 protein and apoptotic Bax gene expressions and increasing anti-apoptotic gene $B C L-x L$. The 3,4,4'-trimethoxy moiety in stilbene structure is an element responsible for the antiproliferative activity. Hydroxylation of DMU-212 metabolites in position 3, 4 and 4' largely lowers antiproliferative properties. The published results point out that the anticancer activity of DMU-212 is primarily a result of metabolic conversion to DMU-214.

The studies conducted by Androutsopoulos et al. [2] show that DMU-281, DMU-291 and DMU-807 have also the ability to inhibit the proliferation of breast 
cancer cells MCF-7 and liver HepG -2, however, one has failed in setting $\mathrm{IC}_{50}$ values for them. The presence of free hydroxyl groups in positions 3, 4 and 4' results in reduced pharmacological activity due to rapid excretion in the form of glucuronides and sulfates, as well as reduced compound lipophilicity.

DMU-212 and its metabolite DMU-214 show pro-apoptotic properties as a result of protein p53 activation, which is a cell cycle inhibitor. As a result of the apoptosis activation, the next cell division does not take place [2]. Apoptosis is the desired type of cancer cell death induced by anti-cancer drugs because it does not lead to the inflammatory process formation.

\section{REFRENCES}

[1] Aldawsari SF, Velazquez-Martinez CA. 3,4',5-trans-Trimethoxystilbene; a natural analogue of resveratrol with enhanced anticancer potency, The Journal of New Anticancer Agents-Springer Science + Business Media New York 2015; doi: 10.1007/s10637-015-0222-x; Published online: 28.02.2015.

[2] Androutsopoulos VP, Ruparelia KC, Papakyriakou A, Filippakis H, TsatsakisAm, Spandidos DA. Anticancer effects of the metabolic products of the resveratrol analogue, DMU-212: structural requirements for potency, Eur. J. Med. Chem. 2011; 46 (6): 2586-95.

[3] Belleri M, Ribatti D, Nicoli S, Cotelli F, Forti L, Vannini V, Stivala LA, Presta M. Antiangiogenic and vascular-targeting activity of the microtubule-destabilizing trans-resveratrol derivative 3,5,4'-trimethoxystibene, Mol. Pharmcol. 2005; 67 (5): 1451-9.

[4] Berbee JF, Wong MC, Wang Y, van der Hoorn JW, Khedoe PP, Dźwieku JB, Mol IM, Hiemstra PS, TsiKas D, Romis JA. Resveratrol protects against atherosclerosis, but does not add to the antiatherogenic effect of atorvastatin, in APOE*3-Leiden.CETP mice, J.Nutr.Biochem. 2013; 24 (8): 1423-30.

[5] Berta GN, Salamone P., Sprio AE, Di Scipio F., Marinos LM, Sapino S., Carlotti ME, Cavalli R., Di CARLO F. Chemoprevention of 7,12-dimethylbenz[a]anthracene (DMBA) - induced oral carcinogenesis in hamster cheekpouch by topical application of resveratrol complexed with 2-hydroxypropyl-betacyclodextrin, Oral Oncol. 2010; 46 (1): 42-8.

[6] Bertelli AA, Das DK. Grapes, wines, resveratrol, and heart health, J. Cardiovasc. Pharmacol. 2009; 54: 468-476.

[7] Cardile V, Chillemi R, Lombardo L, Sciuto S, Spatafora C, Tringali C. Antiproliferative activity of methylated analogues of E- and Z-resveratrol., Z. Naturforsch C. 2007; 62 (3-4): 189-95.

[8] Cardile V, Lombardo L, Spatafora C, Tringali C. Chemo-enzymatic synthesis and cell-growth inhibition activity of resveratrol analogues, Bioorganic Chemistry. 2005; 33 (1): 22-33.

[9] Chang TK, Yeung RK. Effect of trans-resveratrol on 7-benzyloxy-4-trifluoromethylcoumarin O-dealkylation catalyzed by human recombinant CYP3A4 and CYP3A5, Can J. Physiol. Pharmacol. 2001; 79 (3): 220-6.

[10] Cichocki M, Baer-Dubowska W, Wierzchowski M, Murias M, Jodynis-Liebert J. 3,4,5,4'-trans-tetramethoxystilbene (DMU-212) modulates the activation of NF-KB, AP-1, and STAT3 transcription factors in rat liver carcinogenesis induced by initiation-promotion regimen, Mol Cell Biochem. 2014; 391 (1-2): 27-35.

[11] Colin D, Gimazane A, Lizard G., Izard JC, Solary E, Latruffe N, Delmas D. Effects of resveratrol analogs on cell cycle progression, cell cycle associated proteins and 5-fluorouracil sensitivity in human derived colon cancer cells, Int. J. Cancer. 2009; 124 (12): 2780-8.

[12] Cucciolla V, Borriello O, Oliva A, Galletti P, Zappia V, Ragione FD. Resveratrol:From Basic Science to the Clinic, Cell Cycle 2007; 6 (20): 2495-2510.

[13] DEAK M, FALK H. On the chemistry of resveratrol diastereomers, Monat für Chem. 2003; 134: 883-888. 
[14] Deng YH, Alex D, Huang HQ, Wang N, Yu N, Wang YT, Leung GP, Lee SM. Inhibit ion of TNF- $\alpha-$ mediated endothelial cell-monocyte cell adhesion and adhesion molecules expression by the resveratrol derivative, trans-3,5,4'-trimethoxystilbene, Phytother. Res. 2011; 25 (3): 451-7.

[15] Fulda S. Resveratrol and derivatives for the prevention and treatment of cancer, Drug Discover Today. 2010; 15 (17-18): 757-765.

[16] Goldberg DM, Yan J, Soleas GJ. Absorption of three wine-related polyphenols in three different matrices by healthy subjects, Clin. Biochem. 2003; 36: 79-87.

[17] Harikumar KB, Aggarwal BB. Resver atrol: a multitargeted agent for age-associated chronic diseases, Cell Cycle. 2008; 7 (8): 1020-1037.

[18] Juan ME, Pilar Vinardell M, Planas JM. The daily oral administration of high doses of trans-resveratrol to rats for 28 days is not harmful, Journal of Nutrition 2002; 132 (2): 257-260.

[19] Kang SY, Lee JK, Choi O, Young Kim C, Jang JH, Hwang BY, Hong YS. Biosynthesis of methylated resveratrol analogs through the construction of an artificial biosynthetic pathway in E.coli, BMC Biotechnology, 14: 67; Published online 17.07.2014; doi:10.1186/1472-6750-14-6.

[20] Kopeć A, Piątrowska E, Leszczyńska T, Bieżanowska-Kopeć R. Prozdrowotne właściwości resweratrolu, Nauka Technologia Jakość 2011; 5 (78): 5-15.

[21] Kumar A, Negi G, Sharma SS. Neuroprotection by resveratrol in diabetic neuropathy: concepts \& mechanisms, Curr. Med. Chem. 2013; 20 (36): 4640-5.

[22] Lin HS, Ho PC. Preclinical pharmacokinetic evaluation of resveratrol trimethyl ether in Sprague-dawley rats: the impacts of aqeous solubility, dose escalation, food and repeated dosing on oral bioavailability, J. Pharm. Sci. 2011; 100 (10): 4491-500.

[23] Liu Yin MY, Wy X, Zeng M, Zhao Q, Keefe Liu DL. Resveratrol protects against age-associated infertility in mice, Hum. Reprod. 2013; 28 (3): 707-717.

[24] MA Z, MOLAVI O, HADDADI A, LAI R, GOSSAGE RA, LAVASANIFAR A. Resveratrol analog trans 3,4,5,4'-tetramethoxystilbene(DMU-212) mediates anti-tumor effects via mechanism different from that of resveratrol, Cancer Chemother. Pharmacol. 2008; 63 (1): 27-35.

[25] Mikstacka R, Rimando AM, Dutkiewicz Z, Stefański T, Sobiak S. Design, synthesis and evaluation of the inhibitory selectivity of novel trans-resveratrol analogues on human recombinant CYP1A1, CYP1A2 and CYP1B1, Bioorganic \& Medicinal Chemistry 2012; 20 (17): 7117-5126.

[26] Okawara M, Katsuki H, Kurimoto E, Shibata H, Kume T. Akaike A. Resveratrol protects dopaminergic neurons in midbrain slice culture from multiple insults, Biochem. Pharmacol. 2007; 73: 550-560.

[27] Olas B. Resweratrol jako dobroczyńca w profilaktyce chorób układu krążenia, Kosmos problemy nauk biologicznych 2006; 55 (2-3): 277-285.

[28] Ovesna Z, Horvathova-Kozics K. Structure-activity relationship of trans-resveratrol and its analogues, Neoplasma2005; 529 (6): 450-5.

[29] Park E-J, Pezzuto JM. The pharmacology of resveratrol in animals and humans, BBA-Molecular Basis of Disease 2015; 1852 (6): 1071-1113.

[30] PERVAIZ S. Resveratrol: from grapevines to mammalian biology, FASEB J. 2003; 17: 1975-1985.

[31] Piotrowska H, Myszkowski K, ZióŁkowska A, Kulcenty K, Wierzchowski M, Kaczmarek M, Murias M, KwiatkowsKa-BorowcZyk E, Jodynis-Liebert J. Resveratrol analogue 3,4,4',5-tetramethoxystilbene inhibits growth, arrests cell cycle and induces apoptosis in ovarian SKOV-3 and A-2780 cancer cells, Toxicol. Apppl. Pharmacol. 2012; 263 (1): 53-60.

[32] Potter Ga, Patterson LH, Wanogho E, Perry PJ, Butler PC, Ijaz T, RupareliaKC,Lamb JH, Farmer $\mathrm{PB}$, StANLEy LA, Burke MD. The cancer preventative agent resveratrol is converted to the anticancer agent piceatannol by the cytochrome P450 enzyme CYP1B, Br. J. Cancer 2002; 86: 774-778.

[33] Rahal K, Schmiedlin-Ren P, Adler J, Dhanani M, Sultani V, Rittershaus AC, Reingold L, McKenNa BJ, Christman GM. Resveratrol has anti-inflammatory and antifibrotic effects in the peptidoglycan-polysaccharide rat model of Crohn's disease, Inflamm Bowel Dis. 2012; 18 (4): 613-23.

[34] Regev-Shoshani G, Shoseyov O, Bilkis I, Kerem Z. Glycosylation of resveratrol protects it from enzymic oxidation, Biochem.J. 2003; 374: 157-163. 
[35] Roberti M, Pizzirani D, Simoni D, Rondanin R, Baruchello R, Bonora C, Buscemi F, Grimaudo $\mathrm{S}$, ToLomeo M. Synthesis and biological evaluation of resveratrol and analogs as apoptosis-inducing agents, J. Med. Chem. 2003; 46: 3546-3554.

[36] Sale S, Tunstall RG, Ruparelia KC, Potter GA, Steward WP, Gescher AJ. Comparison of the effects of the chemopreventive agent resveratrol and its analog trans 3,4,5,4'-tetramethoxystilbene (DMU-212) on adenoma development in the Apc(Min+) mouse and cyclooxygenase-2 in human-derived colon cancer cells, Int. J. Cancer. 2005; 115 (2): 194-201.

[37] Sale S, Verschoyle RD, Boocock D, Jones DJ.L, Wilsher N, Ruparelia KC, Potter Ga, Farmer PB, SteWARD WP, Gescher AJ. Pharmacokinetics in miceand growth-inhibitory properties of the putative cancer chemopreventive agent resveratrol and the synthetic analogue trans 3,4,5,4'-tetramethoxystilbene, Br. J. Cancer 2004; 90 (3): 736-744.

[38] Schraumen P, Timmers S. Can resveratrol help to maintain metabolic helath?, Proc. Nut.r Soc. 2014; 73 (2): 271-7.

[39] Simoni D, Roberti M, Invidiata FP, Aiello E, Aiello S, Marchett P. Stilbene-based anticancer agents: Resveratrol analogues active toward HL-60 leukemic cells with a non-specific phas mechanism., Bioorg. Med. Chem. Lett. 2006; 16 (12): 3245-3248.

[40] Singh N, Agrawal M, Dore S. Neuroprotective properties and mechanisms of resveratrol in in vitro and in vivo experimental cerebral stroke models., ACS Chem. Neurosci. 2013; 4 (8): 1151-1162.

[41] Sun X. Measurement and correlation of stability of trans-resveratrol in 11 solvents at $\mathrm{T}=(278,2,288.2$, 298.2 and 318.2), K. J. Chem. Thermodynamics 2008; 40: 735- 738.

[42] Szaefer H, Cichocki M, Majchrzak-Celińska A. Nowe cytochromy P450 jako biomarkery i potencjalne cele oddziaływania w chemio prewencji i terapii nowotworów, PostepyHig. Med. Dosw. (online) 2013; 67: 709-718.

[43] Udenigwe CC, Ramprasath VR, Aluko RE. Jones P.JH. Potential of resveratrol in anticancer and anti-inflammatory therapy, Nutrition Reviews 2008; 66 (8): 445-454.

[44] Waffo-Teguo P, Hawthorne ME, Cuendet M, Merillon JM, Kinghorn AD, Pezzuto JM, Mehta RG. Potential cancer - chemopreventive activities of wine stilbenoids and flavans extracted from grape (Vitisvinifera) cell cultures, Nutr. Cancer. 2001; 40: 173.

[45] Wenezel E, Somoza V. Metabolism and bioavailability of trans-resveratrol, Mol.Nutr.Food Res. 2005; 49: 472-481.

[46] You-QiuXue, Jin-Ming Di, Yun Luo, Ke-Jun Cheng, Xing Wei, Zhi Shi. Resveratrol oligomers for prevention and treatment of cancers, Oxid Med Cell Longev. 2014, Published online 2014 March 23.

[47] Yu C, Shin YG, Kosmeder JW, Pezzuto JM, van Breemen RB. Liquid chromatography/tandem mass spectrometric determination of inhibition of human cytochrome P450 isozymes by resveratrol and resveratrol-3-sulfate, Rapid Commun. Mass Spectrom. 2003; 17 (4): 307-13.

[48] Zdrojewicz Z, Belowska-Bień K. Resweratrol - działanie i znaczenie kliniczne, Adv. Clin. Exp. Med. 2005; 14 (5): 1051-1056.

Bartosz Kempisty

Department of Histology and Embryology, Department of Anatomy

Poznań University of Medical Sciences

6 Święcickiego St., 60-781 Poznań, Poland

tel./fax: $+48618546418 /+48618546440$

e-mail: bkempisty@ump.edu.pl 